\title{
Optimal generalized Heronian mean bounds for the logarithmic mean
}

\author{
Hong-Xing Shi ${ }^{1}$, Bo-Yong Long ${ }^{2}$ and Yu-Ming $\mathrm{Chu}^{3^{*}}$
}

\author{
* Correspondence: \\ chuyuming2005@yahoo.com.cn \\ ${ }^{3}$ School of Mathematics and \\ Computational Science, Hunan City \\ University, Yiyang 413000, China \\ Full list of author information is \\ available at the end of the article
}

\begin{abstract}
In this article, we establish a double inequality between the generalized Heronian and logarithmic means. The achieved result is inspired by the articles of Lin and Shi et al., and the methods from Janous. The inequalities we obtained improve the existing corresponding results and, in some sense, are optimal.
\end{abstract}

2010 Mathematics Subject Classification: 26E60.

Keywords: generalized Heronian mean, logarithmic mean, power mean

\section{Introduction}

The logarithmic mean $L(a, b)$ of two positive numbers $a$ and $b$ is defined by

$$
L(a, b)= \begin{cases}\frac{a-b}{\log a-\log b}, & a \neq b, \\ a, & a=b .\end{cases}
$$

Recently, the logarithmic mean has been the subject of intensive research. In particular, many remarkable inequalities for the logarithmic mean can be found in the literature [1-30]. It might be surprising that the logarithmic mean has applications in physics, economics, and even in meteorology [31-33]. In [31], Kahlig and Matkowski study a variant of Jensen's functional equation involving the logarithmic mean, which appears in a heat conduction problem. A representation of the logarithmic mean as an infinite product and an iterative algorithm for computing the logarithmic mean as the common limit of two sequences of special geometric and arithmetic means are given in [17]. In [34,35] it is shown that the logarithmic mean can be expressed in terms of Gauss's hypergeometric function ${ }_{2} F_{1}$. And, in [35], Carlson and Gastafson prove that the reciprocal of the logarithmic mean is strictly totally positive, that is, every $n \times n$ determinant with elements $\frac{1}{L\left(x_{i}, y_{i}\right)}$, where $0<x_{1}<x_{2}<\ldots<x_{n}$ and $0<y_{1}<y_{2}<\ldots<y_{n}$ are positive for all $n \geq 1$.

The power mean of order $r$ of two positive numbers $a$ and $b$ is defined by

$$
M_{r}(a, b)= \begin{cases}\left(\frac{a^{r}+b^{r}}{2}\right)^{\frac{1}{r}} & r \neq 0, \\ \sqrt{a b}, & r=0 .\end{cases}
$$

It is well-known that $M_{r}(a, b)$ is continuous and strictly increasing with respect to $r \in \mathbb{R}$ for fixed $a, b>0$ with $a \neq b$.

(C) 2012 Shi et al; licensee Springer. This is an Open Access article distributed under the terms of the Creative Commons Attribution License (http://creativecommons.org/licenses/by/2.0), which permits unrestricted use, distribution, and reproduction in any medium, provided the original work is properly cited. 
Lin [18] presents the sharp power mean bounds for logarithmic mean as follows:

$$
M_{0}(a, b)<L(a, b)<M_{\frac{1}{3}}(a, b)
$$

for all $a, b>0$ with $a \neq b$.

For $\omega \geq 0$ and $p \in \mathbb{R}$ the generalized Heronian mean $H_{p, \omega}(a, b)$ of two positive numbers $a$ and $b$ is introduced by Shi et al. [36] as follows:

$$
H_{p, \omega}(a, b)= \begin{cases}{\left[\frac{a^{p}+\omega(a b)^{\frac{p}{2}}+b^{p}}{\omega+2}\right]^{\frac{1}{p}},} & p \neq 0 . \\ \sqrt{a b}, & p=0 .\end{cases}
$$

From (1.2) and (1.4) we clearly see that $H_{p, 0}(a, b)=M_{p}(a, b)$ for all $p \in \mathbb{R}$ and $a, b$ $>0$. It easily follows from (1.4) that $H_{p, \omega}(a, b)$ is continuous with respect to $p \in \mathbb{R}$ for fixed $a, b>0$ and $\omega \geq 0$, strictly increasing with respect to $p \in \mathbb{R}$ for fixed $a, b>0$ with $a \neq b$ and $\omega \geq 0$, strictly decreasing with respect to $\omega \geq 0$ for fixed $a, b>0$ with $a \neq b$ and $p>0$, and strictly increasing with respect to $\omega \geq 0$ for fixed $a, b>0$ with $a \neq$ $b$ and $p<0$.

In [37], Janous prove that

$$
L(a, b)<H_{1,4}(a, b)
$$

for all $a, b>0$ with $a \neq b$.

The purpose of this article is to find the greatest value $p=p(\omega)$ and the least value $q=q(\omega)$ such that the double inequality $H_{p, \omega}(a, b)<L(a, b)<H_{q, \omega}(a, b)$ holds for fixed $\omega \geq 0$ and all $a, b>0$ with $a \neq b$.

\section{Main result}

Theorem 2.1. For fixed $\omega \geq 0$ and all $a, b>0$ with $a \neq b$ we have

$$
H_{0, \omega}(a, b)<L(a, b)<H_{\frac{\omega+2}{6}, \omega}(a, b),
$$

and $H_{\frac{\omega+2}{6}, \omega}(a, b)$ and $H_{0, \omega}(a, b)$ are the best possible upper and lower generalized Heronian mean bounds of the logarithmic mean $L(a, b)$, respectively.

Proof. Without loss of generality, we assume $a>b$ and put $t=\frac{a}{b}>1$. Then from (1.1) and (1.4) we get

$$
\begin{aligned}
& \log [L(a, b)]-\log \left[H_{\frac{\omega+2}{6}, \omega}(a, b)\right] \\
& =\log \left(\frac{t-1}{\log t}\right)-\frac{6}{\omega+2} \log \left(\frac{1+\omega t^{\frac{\omega+2}{12}}+t^{\frac{\omega+2}{6}}}{\omega+2}\right) .
\end{aligned}
$$

Let

$$
f(t)=\log \left(\frac{t-1}{\log t}\right)-\frac{6}{\omega+2} \log \left(\frac{1+\omega t^{\frac{\omega+2}{12}}+t^{\frac{\omega+2}{6}}}{\omega+2}\right) .
$$


Then simple computations lead to

$$
\begin{aligned}
& \lim _{t \rightarrow 1+} f(t)=0, \\
& f^{\prime}(t)=\frac{f_{1}(t)}{t(t-1)\left(1+\omega t^{\frac{\omega+2}{12}}+t^{\frac{\omega+2}{6}}\right) \log t},
\end{aligned}
$$

where $f_{1}(t)=\left(\frac{\omega}{2} t^{\frac{\omega+14}{12}}+t^{\frac{\omega+2}{6}}+\frac{\omega}{2} t^{\frac{\omega+2}{12}}+t\right) \log t-t^{\frac{\omega+8}{6}}+t^{\frac{\omega+2}{6}}-\omega t^{\frac{\omega+14}{12}}+\omega t^{\frac{\omega+2}{12}}-t+1$.

$$
\begin{aligned}
f_{1}(1) & =0 \\
f^{\prime}(t) & \left.=\left[\frac{\omega(\omega+14)}{24} t^{\frac{\omega+2}{12}}+\frac{\omega(\omega+2)}{24} t^{\frac{\omega+10}{12}}+\frac{\omega+2}{6} t^{\frac{\omega+4}{6}}+1\right]\right] \log t \\
- & \frac{\omega+8}{6} t^{\frac{\omega+2}{6}}+\frac{\omega+8}{6} t^{\frac{\omega+4}{6}}-\frac{\omega(\omega+8)}{12} t^{\frac{\omega+2}{12}}+\frac{\omega(\omega+8)}{12} t^{\frac{\omega-10}{12}}
\end{aligned}
$$

$$
f_{1}^{\prime}(1)=0,
$$

$$
f_{1}^{\prime \prime}(t)=\frac{1}{288} t^{\frac{\omega-10}{6}} f_{2}(t)
$$

where $\begin{aligned} & f_{2}(t)=\left[\omega(\omega+2)(\omega+14) t^{\frac{10-\omega}{12}}+\omega(\omega+2)(\omega-10) t^{\frac{\omega+2}{12}}+8(\omega+2)(\omega-4)\right] \log t-2 \omega\left(\omega^{2}+\right. \\ & 4 \omega-68) t^{\frac{10-\omega}{12}}+2 \omega\left(\omega^{2}+4 \omega-68\right) t^{\frac{\omega+2}{12}}+288 t^{\frac{4-\omega}{6}}-8(\omega+2)(\omega+8) t+8\left(\omega^{2}+10 \omega-20\right)\end{aligned}$

$$
\begin{aligned}
& f_{2}(1)=0, \\
& f_{2}^{\prime}(t)=\frac{1}{12} t^{-\frac{\omega+14}{12}} f_{3}(t),
\end{aligned}
$$

where $\begin{aligned} & f_{3}(t)=-\left[\omega(\omega+2)(\omega+14)(\omega-10) t-\omega(\omega+2)^{2}(\omega-10)\right] \log t+2 \omega\left(\omega^{3}-12 \omega+848\right) t+ \\ & 2 \omega(\omega+2)^{2}(4-\omega)+96(\omega+2)(\omega-4) t^{\frac{\omega+12}{12}}-576(\omega-4) t^{\frac{10-\omega}{12}}-96(\omega+2)(\omega+8) t^{\frac{\omega+14}{12}} .\end{aligned}$

$$
f_{3}(1)=0 \text {, }
$$

$$
\begin{aligned}
f^{\prime}{ }_{3}(t) & =-\omega(\omega+2)(\omega+14)(\omega-10) \log t-\omega(\omega+2)^{2}(\omega-10) t^{-1} \\
& +8(\omega+2)^{2}(\omega-4) t^{\frac{\omega+10}{12}}-8(\omega+2)(\omega+8)(\omega+14) t^{\frac{\omega+2}{12}} \\
& +48(\omega-4)(\omega-10) t^{\frac{\omega+2}{12}}+\omega\left(\omega^{3}-6 \omega^{2}+108 \omega+1976\right)
\end{aligned}
$$

$$
f_{3}^{\prime}(1)=0,
$$

$$
f_{3}^{\prime \prime}(t)=t^{-2} f_{4}(t)
$$

where

$$
f_{4}(t)=-\omega(\omega+2)(\omega+14)(\omega-10) t+\omega(\omega+2)^{2}(\omega-10)+\frac{2}{3}(\omega+2)^{2}(\omega-4)(\omega-
$$

$10) t^{\frac{\omega+2}{12}}-\frac{2}{3}(\omega+2)^{2}(\omega+8)(\omega+14) t^{\frac{\omega+14}{12}}-4(\omega-2)(\omega-4)(\omega-10) t^{\frac{10-\omega}{12}}$.

$$
f_{4}(1)=-8(\omega+2)\left(5 \omega^{2}-10 \omega+32\right)<0,
$$




$$
\begin{aligned}
f_{4}^{\prime}(t) & =-\omega(\omega+2)(\omega+14)(\omega-10)+\frac{1}{18}(\omega+2)^{3}(\omega-4)(\omega-10) t^{\frac{\omega-10}{12}} \\
& -\frac{1}{18}(\omega+2)^{2}(\omega+8)(\omega+14)^{2} t^{\frac{\omega+2}{12}} \\
& +\frac{1}{3}(\omega+2)(\omega-4)(\omega-10)^{2} t^{-\frac{\omega+2}{12}} \\
f_{4}^{\prime}(1) & =-\frac{2}{3}(\omega+2)\left(5 \omega^{3}+60 \omega^{2}-108 \omega+448\right)<0, \\
f_{4}^{\prime \prime}(t)= & \frac{1}{216}(\omega+2)^{2} t^{-\frac{\omega+14}{12}} f_{5}(t)
\end{aligned}
$$

where $\begin{gathered}f_{5}(t)=(\omega+2)(\omega-4)(\omega-10)^{2} t^{\frac{\omega-4}{6}}-(\omega+2)(\omega+8)(\omega+14)^{2} t^{\frac{\omega-2}{6}}-6(\omega-\text {. } \\ 4)(\omega-10)^{2}\end{gathered}$

$$
\begin{aligned}
& f_{5}(1)=-6\left(11 \omega^{3}+36 \omega^{2}+588 \omega+256\right)<0, \\
& f_{5}^{\prime}(t)=\frac{\omega+2}{6} t^{\frac{\omega-10}{6}} f_{6}(t)
\end{aligned}
$$

where

$$
\begin{aligned}
& f_{6}(t)=(\omega-4)^{2}(\omega-10)^{2}-(\omega+2)(\omega+8)(\omega+14)^{2} t \\
& f_{6}(1)=-6\left(11 \omega^{3}+36 \omega^{2}+588 \omega+256\right)<0
\end{aligned}
$$

From (2.22) we clearly see that $f_{6}(t)$ is strictly decreasing in $[1,+\infty)$, then $(2.23)$ leads to that

$$
f_{6}(t)<0
$$

for $t \in(1,+\infty)$.

It easily follows from (2.5)-(2.21) and (2.24) that

$$
f^{\prime}(t)<0
$$

for $t \in(1,+\infty)$.

Therefore, $L(a, b)<H_{\frac{\omega+2}{6}, \omega}(a, b)$ follows from (2.2)-(2.4) and (2.25).

On the other hand, $H_{0, \omega}(a, b)=M_{0}(a, b)<L(a, b)$ follows from (1.3).

Next, we prove that $H_{0, \omega}(a, b)$ and $H_{\frac{\omega+2}{6}, \omega}(a, b)$ are the optimal lower and upper generalized Heronian mean bounds of the logarithmic mean $L(a, b)$.

For any $0<\varepsilon<\frac{\omega+2}{6}, \omega \geq 0$ and $x>0$, from (1.1) and (1.4) we have

$$
\begin{aligned}
& \lim _{x \rightarrow+\infty} \frac{H_{\varepsilon, \omega}(1, x)}{L(1, x)} \\
& \quad=(\omega+2)^{-\frac{1}{\varepsilon}} \lim _{x \rightarrow+\infty}\left[\frac{\left(1+\omega x^{-\frac{\varepsilon}{2}}+x^{-\varepsilon}\right)^{\frac{1}{\varepsilon}}}{1-\frac{1}{x}} \log x\right] \\
& =+\infty
\end{aligned}
$$




$$
\begin{aligned}
& \log [L(1,1+x)]-\log \left[H_{\frac{\omega+2}{6}-\varepsilon, \omega}(1,1+x)\right] \\
& =\frac{\varepsilon}{4(\omega+2)} x^{2}+o\left(x^{3}\right) \quad(x \rightarrow 0)
\end{aligned}
$$

Equations (2.26) and (2.27) imply that for any $\omega \geq 0$ and $0<\varepsilon<\frac{\omega+2}{6}$ there exist sufficiently large $X=X(\varepsilon, \omega)>1$ and sufficiently small $\delta=\delta(\varepsilon, \omega)>0$; such that $H_{\varepsilon, \omega}$ $(1, x)>L(1, x)$ for $x \in(X,+\infty)$ and $L(1,1+x)>H_{\frac{\omega+2}{6}-\varepsilon, \omega}(1,1+x)$ for $x \in(0, \delta)$.

Remark 2.1. If we take $\omega=0$, then inequality (2.1) reduce to inequality (1.3).

Remark 2.2. If we take $\omega=4$, then the upper bound in inequality (2.1) becomes the upper bound in inequality (1.5).

\section{Acknowledgements}

This study was partly supported by the Natural Science Foundation of China (Grant nos. 11071069, 11171307 11171105), the Social Science Foundation of China (Grant no. 10BTJ001), the Natural Science Foundation of Hunan Province (Grant no. 09JJ6003), and the Innovation Team Foundation of the Department of Education of Zhejiang Province (Grant no. T200924).

\section{Author details}

${ }^{1}$ School of Primary Education, Chuxiong Normal University, Chuxiong 675000, China ${ }^{2}$ School of Mathematical Science, Anhui University, Hefei 230039, China ${ }^{3}$ School of Mathematics and Computational Science, Hunan City University, Yiyang 413000, China

\section{Authors' contributions}

$\mathrm{H}-\mathrm{XS}$ provided the main idea in this article. B-YL carried out the proof of inequality (2.1) in this article. Y-MC carried out the optimality proof of inequality (2.2) in this article. All authors read and approved the final manuscript.

\section{Competing interests}

The authors declare that they have no competing interests.

Received: 22 December 2011 Accepted: 13 March 2012 Published: 13 March 2012

\section{References}

1. Xia, W-F, Chu, Y-M, Wang, G-D: The optimal upper and lower power mean bounds for a convex combination of the arithmetic and logarithmic means. Abstr Appl Anal 2010 (2010). Article ID 604804, 10

2. Chu, Y-M, Xia, W-F: Two optimal double inequalities between power mean and logarithmic mean. Comput Math Appl. 60(1), 83-89 (2010). doi:10.1016/j.camwa.2010.04.032

3. Shi, M-Y, Chu, Y-M, Jiang, Y-P: Optimal inequalities among various means of two arguments. Abstr Appl Anal. 2009 Article ID 694394, 10 (2009)

4. Chu, Y-M, Xia, W-F: Inequalities for generalized logarithmic means. J Inequal Appl. 2009, Article ID 763252,7 (2009)

5. Long, B-Y, Chu, Y-M: Optimal inequalities for generalized logarithmic, arithmetic, and geometric means. J Inequal Appl. 2010, Article ID 806825, 10 (2010)

6. Chu, Y-M, Long, B-Y: Best possible inequalities between generalized logarithmic mean and classical means. Abstr Appl Anal. 2010, Article ID 303286, 13 (2010)

7. Qiu, Y-F, Wang, M-K, Chu, Y-M, Wang, G-D: Two sharp inequalities for Lehmer mean, identric mean and logarithmic mean. J Math Inequal. 5(3), 301-306 (2010)

8. Xia, W-F, Chu, Y-M: Optimal inequalities related to the logarithmic, identric, arith-metic and harmonic means. Rev Anal Numér Théor Approx. 39(2), 176-183 (2010)

9. Chu, Y-M, Wang, S-S, Zong, C: Optimal lower power mean bound for the convex combination of harmonic and logarithmic means. Abstr Appl Anal. 2011, Article ID 520648, 9 (2011)

10. Chu, Y-M, Wang, M-K: Optimal inequalities between harmonic, geometric, logarithmic, and arithmetic-geometric means. J Appl Math. 2011, Article ID 618929, 9 (2011)

11. Chu, Y-M, Hou, S-W, Gong, W-M: Inequalities between logarithmic, harmonic, arith-metic and centroidal means. J Math Anal. 2(2), 1-5 (2011)

12. Hu, H-N, Wang, S-S, Chu, Y-M: Optimal upper power mean bound for the convex combination of harmonic and logarithmic means. Pac J Appl Math. 4(1), 35-44 (2011)

13. Qiu, Y-F, Wang, M-K, Chu, Y-M: The sharp combination bounds of arithmetic and logarithmic means for Seiffert's mean. Int J Pure Appl Math. 72(1), 11-18 (2011)

14. Allasia, G, Giordano, C, Pečarić, J: On the arithmetic and logarithmic means with applications to Stirling's formula. Atti Sem Mat Fis Univ Modena. 47(2), 441-455 (1999)

15. Alzer, H: Ungleichungen für Mittelwerte. Arch Math. 47(5), 422-426 (1986). doi:10.1007/BF01189983

16. Burk, F: The geometric, logarithmic, and arithmetic mean inequality. Am Math Monthly. 94(6), 527-528 (1987). doi:10.2307/2322844

17. Carlson, BC: The logarithmic mean. Am Math Monthly. 79, 615-618 (1972). doi:10.2307/2317088

18. Lin, TP: The power mean and the logarithmic mean. Am Math Monthly. 81, 879-883 (1974). doi:10.2307/2319447 
19. Maloney, J, Heidel, J, Pečarić, J: A reverse Hölder type inequality for the logarithmic mean and generalizations. J Austral Math Soc Ser B. 41(3), 401-409 (2000). doi:10.1017/S0334270000011322

20. Pittenger, AO: Inequalities between arithmetic and logarithmic means. Univ Beograd Publ Elektrotehn Fak Ser Mat Fiz. 678-715: 15-18 (1980)

21. Pittenger, AO: The symmetric, logarithmic and power means. Univ Beograd Publ ElektrotehnFak Ser Mat Fiz. 678-715, 19-23 (1980)

22. Sándor, J: On the identric and logarithmic means. Aequationes Math. 40(2-3), 261-270 (1990)

23. Sándor, J: A note on some inequalities for means. Arch Math. 56(5), 471-473 (1991). doi:10.1007/BF01200091

24. Sándor, J: On certain identities for means. Studia Univ Babeș-Bolyai Math. 38(4), 7-14 (1993)

25. Sándor, J: On certain inequalities for means. J Math Anal Appl. 189(2), 602-606 (1995). doi:10.1006/jmaa.1995.1038

26. Sándor, J: On certain inequalities for means II. J Math Anal Appl. 199(2), 629-635 (1996). doi:10.1006/jmaa.1996.0165

27. Sándor, J: On certain inequalities for means III. Arch Math. 76(1), 34-40 (2001). doi:10.1007/s000130050539

28. Stolarsky, KB: The power and generalized logarithmic means. Am Math Monthly. 87(7), 545-548 (1980). doi:10.2307/ 2321420

29. Vamanamurthy, MK, Vuorinen, M: Inequalities for means. J Math Anal Appl. 183(1), 155-166 (1994). doi:10.1006/ jmaa.1994.1137

30. Alzer, H, Qiu, S-L: Inequalities for means in two variables. Arch Math. 80(2), 201-215 (2003). doi:10.1007/s00013-003$0456-2$

31. Kahlig, P, Matkowski, J: Functional equations involving the logarithmic mean. Z Angew Math Mech. 76(7), 385-390 (1996). doi:10.1002/zamm.19960760710

32. Pittenger, AO: The logarithmic mean in $n$ variables. Am Math Monthly. 92(2), 99-104 (1985). do:10.2307/2322637

33. Pólya, G, Szegö, G: Isoperimetric Inequalities in Mathematical Physics. Princeton Uni-versity Press, Princeton. (1951)

34. Carlson, BC: Algorithms involving arithmetic and geometric means. Am Math Monthly. 78, 496-505 (1971). doi:10.2307/ 2317754

35. Carlson, BC, Gastafson, JL: Total positivity of mean values and hypergeometric func-tions. SIAM J Math Anal. 14(2), 389-395 (1983). doi:10.1137/0514030

36. Shi, H-N, Bencze, M, Wu, Sh-H, Li, D-M: Schur convexity of generalized Heronian means involving two parameters. J Inequal Appl 2008 (2008). Article ID 879273, 9

37. Janous, W: A note on generalized Heronian means. Math Inequal Appl. 4(3), 369-375 (2001)

doi:10.1186/1029-242X-2012-63

Cite this article as: Shi et al: Optimal generalized Heronian mean bounds for the logarithmic mean. Journal of Inequalities and Applications 2012 2012:63.

\section{Submit your manuscript to a SpringerOpen ${ }^{\circ}$ journal and benefit from:}

- Convenient online submission

- Rigorous peer review

- Immediate publication on acceptance

- Open access: articles freely available online

- High visibility within the field

- Retaining the copyright to your article

Submit your next manuscript at $\boldsymbol{s p r i n g e r o p e n . c o m ~}$ 